Journal of Motor Behavior, ${ }^{* *},{ }^{* \star \star * \_* * \star *}, 2020$

\title{
Control of a whole-body task with uncertain initial conditions: Application to the upstart on bars
}

\author{
${ }^{1}$ Michael $\mathrm{J}$ Hiley and ${ }^{1}$ Maurice $\mathrm{R}$ Yeadon \\ ${ }^{1}$ School of Sport, Exercise and Health Sciences, Loughborough University, Leicestershire, LE11 3TU, UK
}

\begin{abstract}
The aim was to determine whether operating a feedforward schema for generating movement pattern parameters was more successful than an open loop strategy for coping with uncertain initial conditions. A computer simulation model was used to determine the optimal solutions that maximised the likelihood of performing a successful upstart. Feedforward schema were established between movement pattern parameters and initial angular velocity. The success of modifying a pre-planned movement pattern based on the parameter relationships (feedforward) was compared with optimal solutions unable to adapt (open loop) to initial angular velocity. The open loop solution was successful $28 \%$ and $20 \%$ of the time for a full strength (elite) and weaker gymnast. The feedforward strategy had success rates of $99 \%$ and $96 \%$ respectively.
\end{abstract}

Keywords: gymnastics; simulation modelling; control; optimisation; schema

\section{INTRODUCTION}

The upstart is a fundamental swinging skill in gymnastics used to transfer the gymnast from beneath the bar to a position of support above the bar (Figure 1). The skill is used by elite and novice gymnasts in men's and women's artistic gymnastics, and can be performed on the horizontal bar, the parallel bars and the uneven bars. In order to receive no deductions from the judges (FIG, 2017) the gymnast must show good extension at the front of the swing with straight arms and legs. The technique of an upstart comprises a swing phase to a position of body extension (Figure 1 A-C), the feet are then brought to the bar (Figure $1 \mathrm{D}$ ), followed by a "kip" (Figure $1 \mathrm{D}-\mathrm{E})$ which is used to raise the centre of mass to the bar. At the 2016 Rio Olympic Games a total of 365 upstarts were performed in the qualifying competition by 110 gymnasts, with $100 \%$ successfully transferring the gymnast to a position above the bar. Despite being a fundamental skill, the upstart is often difficult to learn (Gymnastics HQ, 2016) as it requires coordination of the actions at the hip and shoulder with the rotational position (the swing) of the body.

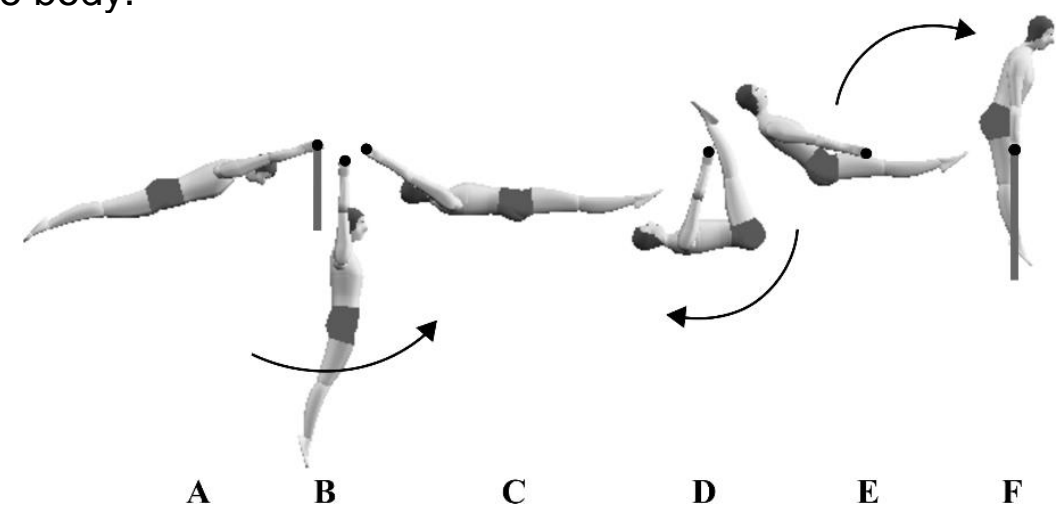

Figure 1. An upstart comprises a swing phase to a position of body extension (A-C), the feet are then brought to the bar (D), followed by a "kip" (D-E) which is used to raise the centre of mass to the bar. 
Previous research has focused on determining the control strategy and solution space used in the upstart using computer simulation modelling (Yamasaki, Gotoh \& Xin, 2010; Hiley \& Yeadon, 2014; Hiley \& Yeadon, 2013). The technique (joint angle time histories at the hip and shoulder) for the upstart has been optimised using various criteria, e.g. minimising angle jerk, joint torque change and effort (Yamasaki et al., 2010). Hiley and Yeadon (2013) compared such criteria with that of maximising success at the task whilst operating in a noisy environment and found that the latter produced solutions most closely replicating gymnastic performances. In the optimisation process noise was added to the joint angle time histories based on measures of human movement variability which were used to represent movement execution noise resulting from the motor system (van Beers, Haggard \& Wolpert, 2004). Success was expressed as the percentage of upstarts able to transport the gymnast to a position above the bar despite the presence of the motor system noise (Hiley \& Yeadon, 2013). In other words, the technique adopted by gymnasts appeared to be characterised by success in ensuring the gymnast reached the final position above the bar.

Despite providing insight to technique and the underlying strategy used by gymnasts, the previous studies have used computer simulation models in an open loop manner (Jagacinski \& Flach, 2003). All simulations within the optimisations described above have started from the same initial conditions. Therefore, no compensation for variable initial conditions or error in the estimation of initial conditions (Miall \& Wolpert, 1996) has been accounted for. Due to the sensitive nature of optimal solutions, it is likely that if the above studies had the initial angular velocity perturbed sufficiently, the solution would no longer be successful, and a new optimal solution would need to be found. Given that the duration of the upstart is over one second, it is likely that gymnasts use some form of either feedforward or feedback control based on an updated evaluation of the initial/current conditions to modify the movement pattern (Jagacinski \& Flach, 2003; Miall \& Wolpert, 1996). As the gymnast swings from the starting position through the lower part of the swing (Figure 1 A-B) there will be time to evaluate the whole-body angular velocity and subsequently select the most appropriate movement pattern to successfully complete the skill.

How the gymnast selects (feedforward) the correct movement pattern would most likely depend on the magnitude of the updated estimate of the whole-body angular velocity (Hiley \& Yeadon, 2016). Based on experience the gymnast learns to adjust the parameters that define the movement pattern in order to perform a successful upstart. Schema theory (Schmidt, 1975) proposed that learning took the form of abstracting relationships between initial conditions, the parameters that define the movement and the movement outcome. Schmidt (2003) defines a schema as "a rule developed by practice and experience which describes a relationship between the outcomes achieved on past attempts at running a program and the parameters chosen on those attempts", where program refers to the concept of a centrally stored set of motor commands (Schmidt, 1975). While there has been debate over the schema theory of motor learning (e.g. Schmidt, 2003; Newell, 2003; Shea \& Wulf, 2005), particularly from a dynamical systems perspective (Newell, 2003), aspects of the original theory are still appealing. It has been shown in certain movements that altering the overall duration leads to a scaling of the movement patterns (Summers, 1975; Shapiro, 1977; Lai, Shea, Wulf \& Wright, 2000; Wulf, Lee \& Schmidt, 1994) so that the relative timing of movements within a sequence is maintained. The technique of the upstart contains invariant features, which are a predetermined set of actions at the hip and shoulder. The initial angular velocity of the upstart can also determine the amplitude of the swing (Figure $1 \mathrm{~A}-\mathrm{C}$ ), and therefore the overall duration of the 
movement. Using schema to scale the timing and magnitude of the hip and shoulder actions based on initial velocity/amplitude of the swing seems to be a reasonable strategy.

The aim of the present study is to determine to what extent operating a feedforward schema for movement pattern parameters based on the initial angular velocity in the upstart is more successful than operating with an open loop strategy (i.e. no modification for error in estimation of initial conditions). Two schemas, based on linear fits and scaled timing of the movement pattern parameters, will be compared to two open loop strategies, both robust to execution errors with one optimised to be robust to initial angular velocity. It is hypothesised that including a variable initial condition when optimising with an open loop approach will lead to a more robust solution than any fixed initial condition optimisation. However, it is expected that operating in a feedforward manner where parameters can be modified based on the initial angular velocity will be more successful.

\section{METHODS}

An angle driven computer simulation model of a gymnast and bar was used to determine the optimal solutions that maximise the likelihood of performing a successful upstart (one that reaches the final support position) from a range of initial angular velocities, while operating with execution noise (van Beers et al., 2004; Hiley \& Yeadon, 2013). Relationships between the movement pattern (joint angle history) parameters and the initial angular velocity were established. The success of modifying a pre-planned movement based on the established parameter relationships (feedforward) was compared with that of optimal solutions that do not adapt (open loop).

\section{Simulation model}

The four-segment planar model of Hiley and Yeadon (2003) which replicates a gymnast and bar was used to simulate the upstart. The bar and gymnast model comprised arm, torso, thigh and lower leg segments. Damped linear springs were used to represent the stretch in the bar and gymnast's shoulder structure (Figure 2). In addition to the stretch in the gymnast, the torso segment was allowed to lengthen as the shoulder elevation angle increased (Begon, Weiber \& Yeadon, 2008).

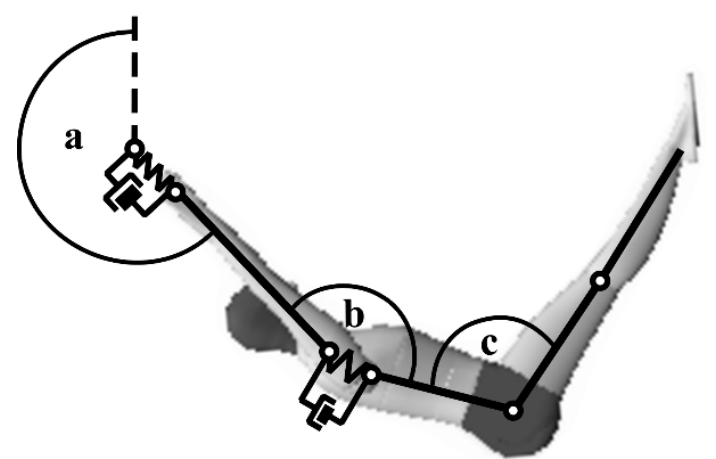

Figure 2. The four-segment simulation model with definition of (a) the arm angle, (b) shoulder angle and (c) hip angle.

Model input comprised segmental inertia parameters, coefficients of the bar and shoulder springs, the initial orientation and angular velocity of the arm and the joint angle time histories at the hip and shoulder in the form of quintic splines (Hiley \& Yeadon, 2013). Output from the model included the time histories of the bar displacement and arm angle (the angle from the vertical of the line joining the neutral 
bar position to the shoulder centre). The arm angle was used to represent the model orientation as the bar to mass centre angle becomes problematic when approaching the support phase. Model parameters were taken from Hiley and Yeadon (2014) in a study of the solution space for an upstart by an elite male gymnast.

\section{Optimisations}

All optimisations were carried out using a genetic optimisation algorithm (Carroll, 2001 ) to maximise the number of successful simulations. Each simulation was given a score of 1 if successful in reaching the support position and 0 if unsuccessful. Simulations with a final shoulder angle and arm angular velocity outside the range (< $30^{\circ}$ and $>180^{\circ} \mathrm{s}$, respectively) obtained from an elite gymnast recorded performing multiple ( $n=10$ ) upstart trials to support (Hiley et al., 2019) were considered to be unsuccessful. All simulations performed within the optimisations started with an extended configuration and an arm angle of $105^{\circ}$ (i.e. $15^{\circ}$ below the horizontal) and ended once the arm angle was in the vertical position above the bar or had reached its minimum value (i.e. failed to reach the support position).. The initial angular velocity of the arm, which represented the angular velocity of the system since initial configuration was fixed for the first $300 \mathrm{~ms}$, was allowed to vary between $0 \% \mathrm{~s}$ to $120 \% \mathrm{~s}$. The range was chosen to be sufficiently large for the gymnast to notice a difference but not so great that a completely different strategy would have to be adopted. Since initial orientation and configuration would also lead to changes in swing amplitude, the present study focussed on variation in the initial angular velocity. During a simulation the model was allowed to make a number of independent angle changes (flexion and extension) at the hip and shoulder. The optimisation algorithm manipulated 7 time and 7 angle parameters (14 in total) which defined the joint angle time histories at the hip and shoulder. The parameters were the maximum and minimum points from the joint angle time histories (Figure 3), therefore defining the time and amplitude of the joint angle changes. The data set of each time history was transformed between consecutive maxima/minima in both the time and angle dimensions (Hiley \& Yeadon, 2013) and quintic splines were fitted to the joint angle time histories of the new data sets (Wood \& Jennings, 1979). The bounds on the angle and time parameter changes were chosen as $\pm 20^{\circ}$ and $\pm 100 \mathrm{~ms}$. If an optimal solution touched any of the bounds the optimisation was repeated using the solution as a starting point with a new set of bounds as described above. No changes in joint angles were allowed to occur before $300 \mathrm{~ms}$ after the start of the simulation (Figure 3). This period was used to represent the time taken to evaluate the initial angular velocity and plan modifications to the preplanned movements.

All joint angle time histories were constrained using joint torque measurements determined from isovelocity dynamometer measurements for a male National Team gymnast, by fitting a function which expressed maximum voluntary torque in terms of joint angle and angular velocity (Forrester, Yeadon, King \& Pain, 2011). If a simulation produced a joint torque that exceeded the maximal value the simulation was considered to be unsuccessful. Joint angle time histories which resulted in the bar passing through the leg segment were also considered to be unsuccessful.

The optimisation criterion was based on maximising the number of successful upstarts despite operating in a "noisy" environment. For each set of joint angle time history parameters produced by the genetic optimisation algorithm (Carroll, 2001), an initial simulation was run with no perturbations added to the joint angle time history parameters. Subsequently another 499 simulations were run based on the same set of parameters, where random perturbations were added to the time and angle parameters with a normal distribution and specified standard deviation. The standard 
deviations (10 ms and 2.3) were taken from Hiley and Yeadon (2012) and perturbations were added using a random number generator and the method of Hiley and Yeadon (2013). The variability introduced with the perturbations was used to represent movement execution noise (van Beers et al., 2004). The percentage of successful simulations out of the 500 was returned as the score for the set of joint angle time history parameters.

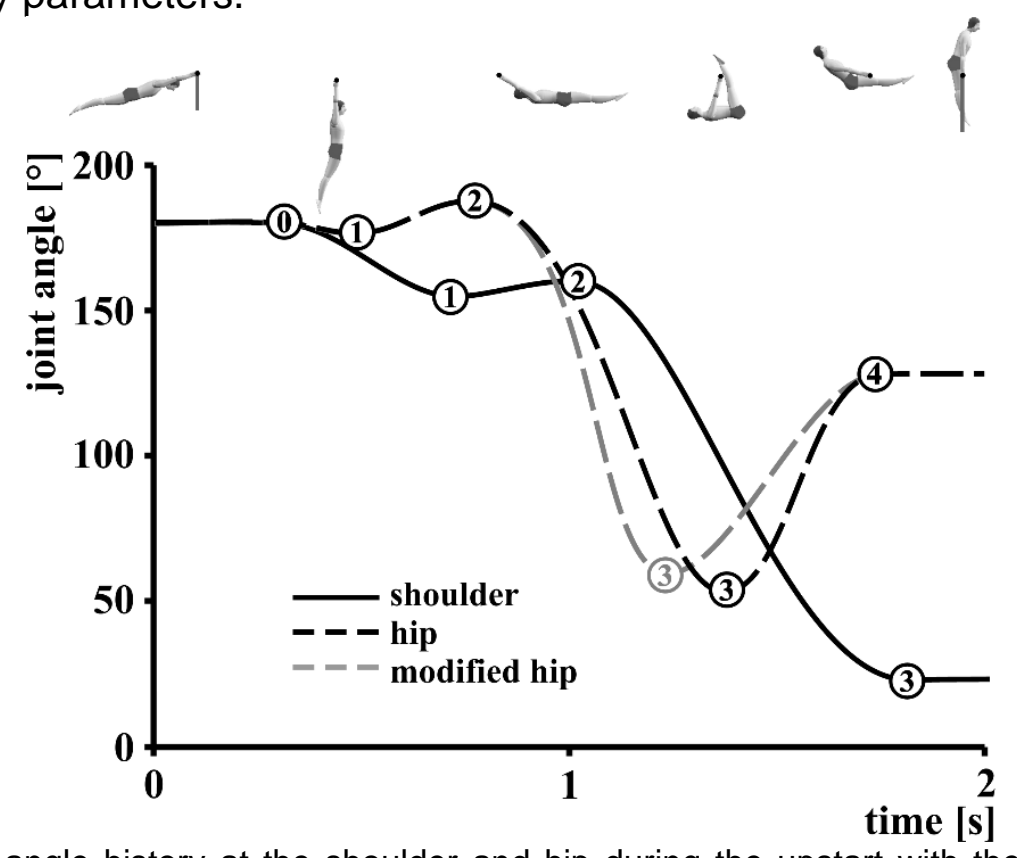

Figure 3. The joint angle history at the shoulder and hip during the upstart with the maximum and minimum points $(1,2,3,4)$ indicated and an example of a modified hip time history for a changed minimum hip angle timing point 3 . Point 0 corresponds to a fixed point in time before which no changes in joint angles occur.

An open loop optimisation for a fixed initial angular velocity of $60 \%$ (mid-range) was carried out. To obtain an open loop solution robust to varying initial angular velocity, an optimisation was performed where the initial angular velocity of each simulation was randomly perturbed between $0 \%$ and $120 \%$ s with a uniform distribution (i.e. all angular velocities equally likely). This process was repeated with strength limits (torque-angular velocity-angle relationships) set to $75 \%$ of maximum to represent a gymnast with less strength than an elite male gymnast. It has previously been shown that a drop in strength of $25 \%$ is sufficient to result in a change in technique and substantially reduce the size of the solution space in which the gymnast can achieve a $90 \%$ plus success rate (Hiley \& Yeadon, 2014).

To obtain data for the schema relating the parameters of the joint angle time histories and the initial angular velocity, 13 individual optimisations were performed with fixed initial angular velocities from $0 \%$ s to $120 \%$ in steps of $10 \%$ s. Again, this process was repeated with strength limits set to $75 \%$ of maximum.

\section{Schema}

Two schemas were developed from the 13 individual optimisations with fixed initial angular velocity. The first schema (schema 1) was based on scaling the time parameters using the overall duration of the upstart. Each time parameter from the 13 optimisations was expressed as a percentage of overall duration. An average percentage for each time parameter was then obtained (Table 1). For a given initial angular velocity the overall duration was determined using the linear fit (Table 2) and the remaining time parameters were expressed as the average percentage of that time. The relationships between initial angular velocity and the angle parameters were 
based on linear fits (Table 2). For the second schema (schema 2) linear relationships were fit between the initial angular velocity and each of the joint angle time history parameters (Table 2, Figure 4). Similar schemas were obtained for the $75 \%$ strength solutions (Tables 1 \& 2).

Table 1. The average ( \pm standard deviation) percentage of overall upstart duration for each joint angle history time parameter across the 13 optimal solutions

\begin{tabular}{|c|c|c|c|c|c|c|c|}
\hline \multirow{3}{*}{ Strength } & \multicolumn{7}{|c|}{ Percentage of overall duration [\%] } \\
\hline & \multicolumn{3}{|c|}{ shoulder } & \multicolumn{4}{|c|}{ hip } \\
\hline & (1) & (2) & (3) & (1) & (2) & (3) & (4) \\
\hline $100 \%$ & $20.5 \pm 0.9$ & $29.0 \pm 2.6$ & $96.1 \pm 0.8$ & $22.8 \pm 1.1$ & $34.5 \pm 2.3$ & $68.8 \pm 1.2$ & $90.7 \pm 1.8$ \\
\hline $75 \%$ & $21.7 \pm 1.6$ & $34.3 \pm 1.6$ & $89.4 \pm 1.6$ & $20.8 \pm 0.9$ & $28.9 \pm 2.3$ & $65.7 \pm 1.3$ & $88.1 \pm 1.1$ \\
\hline
\end{tabular}

Table 2. Linear fits between the initial angular velocity and the optimal joint angle history parameters (times and angles) and overall duration of the upstart

\begin{tabular}{ccccccccc}
\hline & \multicolumn{5}{c}{ Time [s] } & \multicolumn{7}{c}{ Angle [ $\left.{ }^{\circ}\right]$} \\
Parameter & coefficient & constant & $\mathrm{R}^{2}$ & $\mathrm{SE}^{*}$ & coefficient & constant & $\mathrm{R}^{2}$ & $\mathrm{SE}$ \\
\hline 100\% strength & & & & & & & & \\
Shoulder (1) & -0.000 & 0.467 & 0.424 & 0.018 & -0.016 & 181.1 & 0.024 & 3.9 \\
Shoulder (2) & -0.002 & 0.733 & 0.853 & 0.027 & 0.059 & 175.1 & 0.127 & 5.8 \\
Shoulder (3) & -0.002 & 2.183 & 0.891 & 0.022 & 0.069 & 16.1 & 0.725 & 1.6 \\
Hip (1) & -0.001 & 0.542 & 0.734 & 0.018 & -0.010 & 191.3 & 0.008 & 4.0 \\
Hip (2) & -0.002 & 0.853 & 0.926 & 0.018 & -0.020 & 197.3 & 0.017 & 5.7 \\
Hip (3) & -0.002 & 1.596 & 0.976 & 0.010 & -0.081 & 74.1 & 0.666 & 2.1 \\
Hip (4) & -0.001 & 2.002 & 0.668 & 0.017 & 0.132 & 136.2 & 0.340 & 6.9 \\
Duration & -0.002 & 2.265 & 0.980 & 0.009 & & & & \\
75\% strength & & & & & & & & \\
Shoulder (1) & -0.000 & 0.475 & 0.001 & 0.032 & 0.031 & 179.0 & 0.056 & 4.8 \\
Shoulder (2) & -0.001 & 0.819 & 0.805 & 0.022 & -0.010 & 178.3 & 0.012 & 3.5 \\
Shoulder (3) & -0.002 & 2.095 & 0.933 & 0.024 & 0.050 & 18.1 & 0.313 & 2.8 \\
Hip (1) & -0.000 & 0.476 & 0.365 & 0.019 & -0.072 & 190.7 & 0.237 & 4.9 \\
Hip (2) & -0.002 & 0.726 & 0.805 & 0.029 & -0.027 & 186.6 & 0.028 & 5.9 \\
Hip (3) & -0.002 & 1.542 & 0.961 & 0.013 & -0.004 & 66.8 & 0.003 & 2.9 \\
Hip (4) & -0.001 & 1.997 & 0.838 & 0.021 & 0.102 & 132.8 & 0.359 & 5.1 \\
Duration & -0.002 & 2.290 & 0.970 & 0.012 & & & & \\
\hline
\end{tabular}

* Note: SE refers to the standard error of the fit 


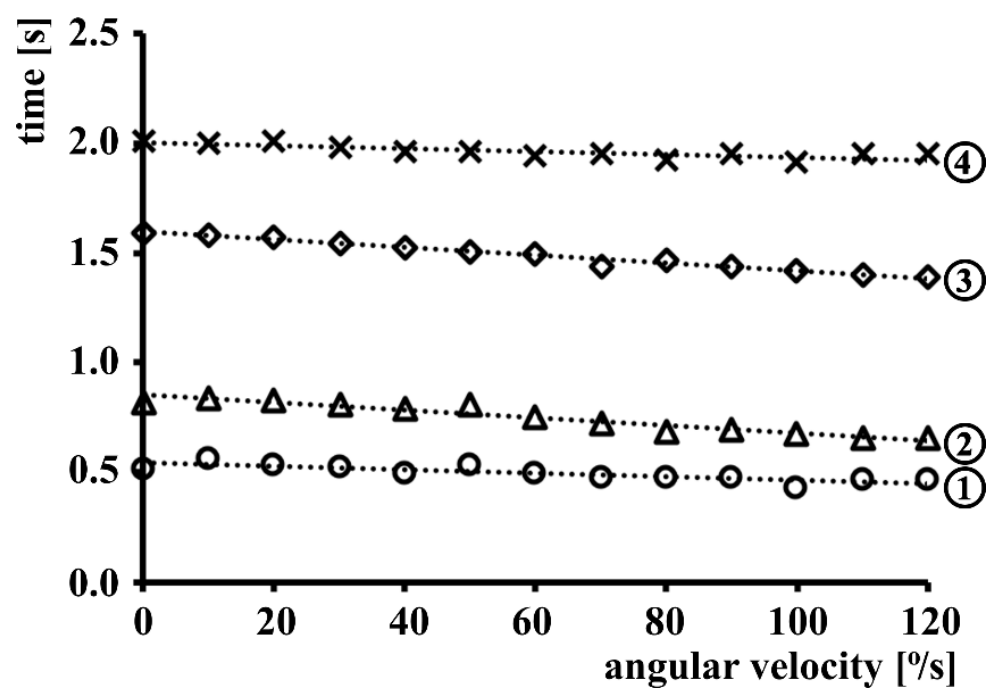

Figure 4. An example of the linear fits between the initial angular velocity and the joint angle history parameters. This example is for the hip timing parameters of the $100 \%$ strength optimisations.

\section{Simulations/Comparisons}

The optimal solutions for the fixed initial angular velocity of $60 \%$ s (open loop), variable initial angular velocity (open loop) and the schemas (feedforward) were run to determine the percentage of successful upstarts $(n=500)$ when operating with a random initial angular velocity (uniform distribution ranging between $0 \% \mathrm{~s}$ and $120 \% \mathrm{~s}$ ). The optimal solution for the fixed initial angular velocity of $60 \% \mathrm{~s}$ was chosen to represent the average technique not accounting for variable initial angular velocity, as it was midway through the range of initial angular velocities used.

\section{RESULTS}

The optimal solutions for the fixed initial angular velocity (open loop) ranging from $0 \%$ to $120 \%$ s produced average $(n=13)$ success rates of $100 \%$ and $99 \%$ for $100 \%$ strength and $75 \%$ strength respectively. When the initial angular velocity was varied between $0 \% \mathrm{~s}$ and $120 \% \mathrm{~s}$ and applied to the solution for a fixed initial angular velocity of $60 \%$, the success rates dropped to $21 \%$ and $16 \%$ respectively (Table 3 ). The solution where the initial angular velocity was allowed to vary (between $0 \% \mathrm{~s}$ and $120 \%$ s) during the optimisation procedure (open loop) was only marginally more successful with $28 \%$ and $20 \%$ for $100 \%$ strength and $75 \%$ strength respectively (Table 3).

Table 3. The success rates of the optimal solutions and schema when attempting to cope with variable initial angular velocity

\begin{tabular}{lcc}
\hline \multirow{2}{*}{ Simulation } & \multicolumn{2}{c}{ Success rate [\%] } \\
\cline { 2 - 3 } & $\begin{array}{c}100 \% \\
\text { strength }\end{array}$ & $\begin{array}{c}75 \% \\
\text { strength }\end{array}$ \\
\hline Open loop (60\%) & $21 \%$ & $16 \%$ \\
Open loop (variable angular velocity) & $28 \%$ & $20 \%$ \\
Schema 1 (overall duration) & $44 \%$ & $40 \%$ \\
Schema 2 & $99 \%$ & $96 \%$ \\
\hline
\end{tabular}


For the first schema (schema 1) where the time parameters of the joint angle histories were expressed as a percentage of the overall duration of the upstart the success rates were $44 \%$ and $40 \%$ for the two strength conditions (Table 3 ). For the second schema (schema 2) where all the joint angle history parameters were fit with linear equations (Table 2), the success rates rose to $99 \%$ and $96 \%$ for the two strength conditions (Table 3 ).

When comparing the 13 solutions for the two strength conditions (Figure 5) the full strength solutions brought the feet to the bar (hip flexion) with a greater average angular velocity $(171 \% \mathrm{~s} \pm 2 \% \mathrm{~s}$ vs $148 \% \mathrm{~s} \pm 2 \%$, paired $\mathrm{t}$ test: $\mathrm{t}=11.1, \mathrm{p}<0.001)$ and subsequently extended with a greater average angular velocity $(158 \%$ s $\pm 3 \%$ s vs $148 \%$ s $\pm 1 \% \mathrm{~s}, \mathrm{t}=3.6, \mathrm{p}=0.003$ ) than the $75 \%$ strength solutions. However, the average angular velocity of closing the shoulder angle (Figure 5) was greater for the $75 \%$ strength solutions $(109 \% \pm 2 \%$ vs $130 \% \pm 1 \%$, paired t test: $t=-23.4, p<0.001)$. After approximately $60 \%$ of the movement, the arm angle became very consistent across all solutions (Figure 5).
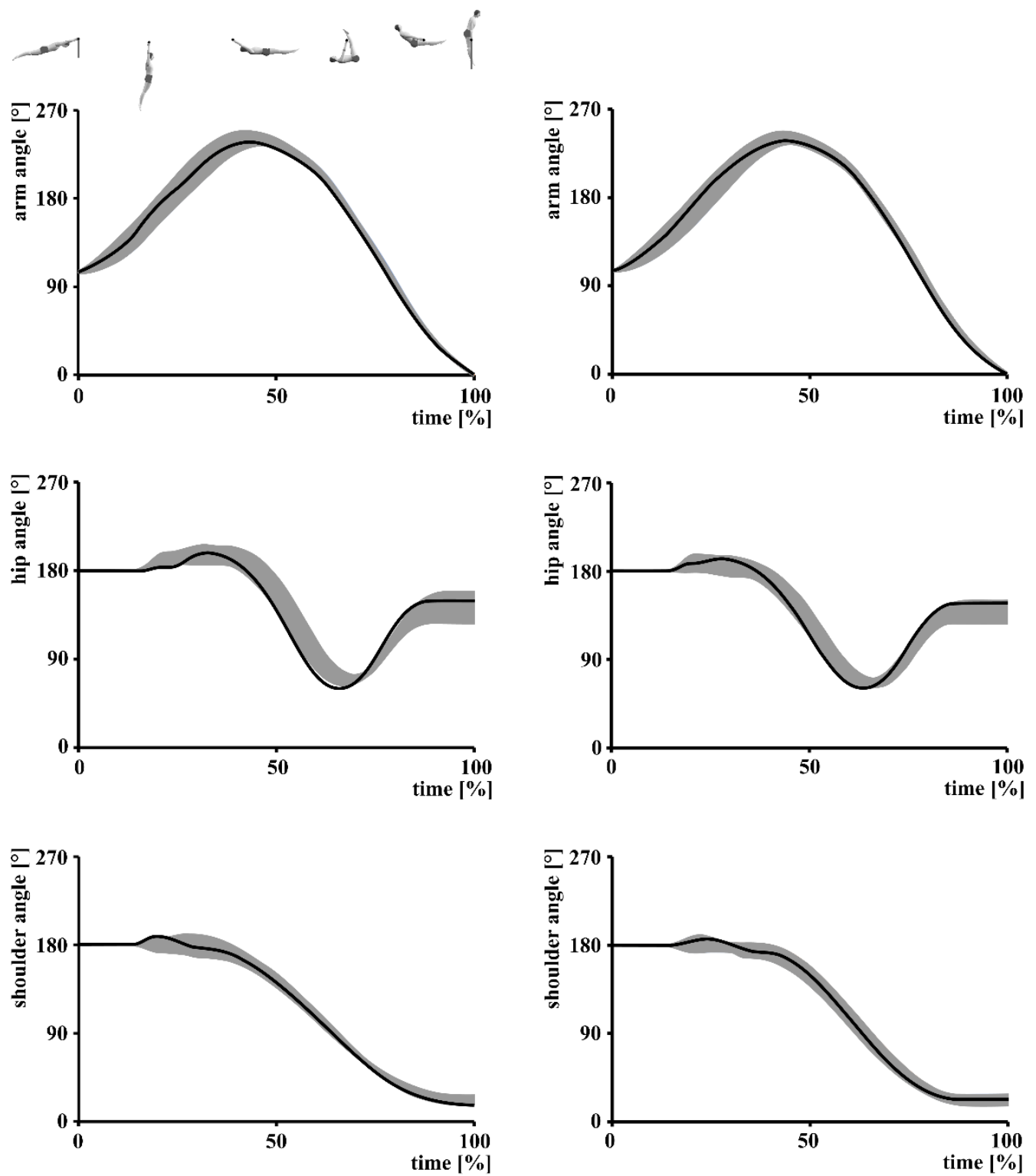

(a)

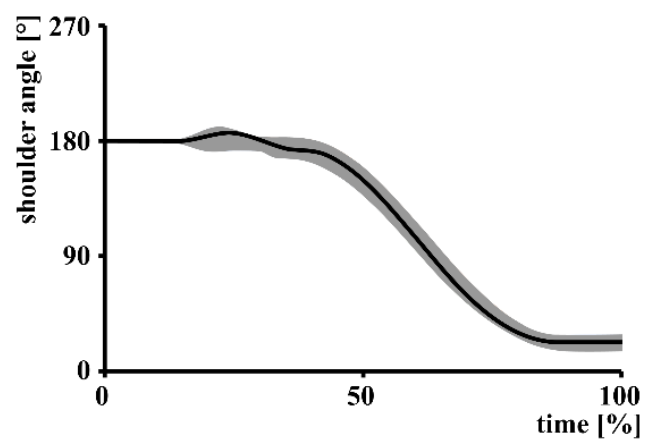

(b)

Figure 5. Joint angle histories for the 13 individual optimal solutions with fixed initial angular velocity ranging from $0 \% \mathrm{~s}$ to $120 \% \mathrm{~s}$ (grey shaded area) and the optimal solution from the variable initial angular velocity optimisations (black line) for (a) $100 \%$ strength and (b) $75 \%$ strength. 


\section{DISCUSSION}

The aim of the study was to determine whether operating a feedforward schema for movement pattern parameters based on the initial angular velocity in the upstart was more successful than operating with an open loop strategy (i.e. no modification for error in estimation of initial conditions). Both schemas (Table 3), developed from optimal solutions with fixed initial angular velocity outperformed the two open loop solutions, suggesting that gymnasts adapt their technique based on the initial angular velocity. Despite carrying out an optimisation specifically to be able to cope with a variable initial angular velocity, operating in an open loop manner was sufficiently unsuccessful (Table 3) compared to elite gymnasts (i.e. Rio Olympics) that it is unlikely to be the strategy adopted. It should be noted that the range of initial angular velocities (resulting from initial orientation and angular velocity) and end point requirements from the Olympic Games (Rio, 2016), will likely be larger than those used in the present study and so the Olympic gymnasts may be selecting from more than one "basic" technique that requires subsequent modification. However, this does not change the conclusion, based on the present study, that an open loop approach is unlikely to be the strategy adopted by gymnasts.

Based on the 13 optimisations with initial angular velocity ranging from $0 \%$ to $120 \%$ s two schema were developed. The first used times scaled from the overall duration of the movement, the second used linear fits to all joint angle time history parameters. While the second schema (schema 2) was very successful, the first schema was surprisingly poor (Table 3 ) and did not come close to the success rate expected for an elite gymnast or one who is regularly able to perform the upstart. This does not follow generalised motor program theory where the relative timing might be expected to be invariant (Schmidt, 1975; Summers, 1975; Shapiro, 1977; Lai et al., 2000; Wulf et al., 1994). One possible explanation for the poor performance might be inferred from the ensemble history of the arm angle (Figure 5). Examining the point by point standard deviations (James, 2004) across the 13 normalised by time data sets there is evidence that the movement is performed in distinct stages. There is a reduction in the joint angle history variance at approximately $70 \%$ of the way through the movement (Figure 5), which corresponds with the "kipping" action (Figure $1 \mathrm{D}-\mathrm{E}$ ). The timing of this action is crucial to raising the centre of mass to the bar. Previous research and coaching literature has highlighted the importance of the timing of the hip and shoulder actions (Bevan \& Corser, 1969; Fukushima \& Russell, 1980; Readhead, 1987; Hiley \& Yeadon, 2013, 2014; Yamasaki et al., 2010), which must be timed with the swing in order to be successful (Hiley et al. 2019). The variability seen in the first half of the skill (Figure 5) may have a functional role in preparation for the kipping action, where functional refers to variation that plays a role in ensuring low variability in the performance outcome (Preatoni, Hamill, Harrison, Hayes, van Emmerik, Wilson \& Rodano, 2013; Hamill, van Emmerik, Heiderscheit \& Li, 1999; Bartlett, Wheat \& Robbins, 2007). The gymnast adjusts the technique, and therefore swing, in order to be in a good place to perform the kip. As a result, the start of the kipping action is performed consistently. The kip is often considered one of the most important aspects of the upstart (Gymnastics HQ, 2016); coaches, however, may wish to pay particular attention to achieving a good starting position for this phase of the movement.

It appears that the upstart may be broken down into distinct phases/actions with adjustments made on this basis, rather than a single adjustment at the start of the swing. When optimising the upstart to determine the underlying strategy Yamasaki et al. (2010) was forced to include via-points in the joint angle time histories to obtain close matches with recorded performances. For the minimum effort optimisation criterion, the via-point corresponded with the start of the kipping action (Yamasaki et 
al., 2010), indicating that achieving this position is indeed important and may be an interim goal. In the present study, the variability in the hip and shoulder angle histories (Figure 5) can be seen to increase prior to the end of the upstart (Figure 5) indicating that some level of control is required in the kipping action to ensure successful completion of the task.

In an experimental perturbation study of targeted movements Grierson and Elliott (2008) found that early control was based on limb velocity comparisons while late control was based on limb and target position. A similar result was obtained for control of the flight phase of a $2 \frac{1}{2}$ somersault forward dive in which early control was based on somersault velocity and subsequent control was based on body orientation (Sayyah, Hiley, King \& Yeadon, 2018). Both studies (Grierson \& Elliott, 2008; Sayyah et al., 2018) suggested that a correction is made for an error in the estimation of the initial conditions and/or pre-planned movement, and that a subsequent correction is made later on in the movement to ensure achieving the end point goal.

One limitation of the present study is that despite determining the success rate of the schemas using variable initial angular velocity, the assumption is that within the $300 \mathrm{~ms}$ where no joint angle changes were permitted, the "gymnast" obtains an accurate evaluation of the current state of the angular velocity and then modifies the technique appropriately. While motor system/execution noise is added to the planned movements, no account is made for the error in the estimation of the initial angular velocity (Wolpert, 1997; Maill \& Wolpert 1996). It is difficult to obtain an accurate measure of a gymnast's ability to estimate angular velocity during swinging as the weighting of the sensorimotor information is unknown, particularly the information from the hand sliding around the bar. Hiley and Yeadon (2016) showed that gymnasts regulated whole-body angular velocity during consecutive long swings on the high bar and were able to control this at the start of the circle to a standard deviation of approximately $2 \%$ s. The evaluation of schema 2 was repeated, with noise added to the initial angular velocity values with a standard deviation of $2 \%$. The noise was added after the joint angle time history parameters had been determined from the schema so as to represent an error in its estimation. The effect on the final success rates of schema 2 (Table 3) were minimal; the 100\% strength evaluation was still $99 \%$ successful, whereas the $75 \%$ strength evaluation dropped $1 \%$ to $95 \%$ successful. Since the schemas were based on solutions generated with execution noise, they also appear to be robust to small errors in the estimation of the angular velocity.

\section{CONCLUSION}

In order to cope with an uncertain initial angular velocity, gymnasts make feedforward adjustments to technique. Although the schema approach was able to achieve high success rates for both strong and weaker gymnasts, it appears that gymnasts may evaluate the state of the performance at more than one instant during the upstart. Modifying all the parameters in advance (feedforward) worked well, but relies on multiple relationships between initial conditions and movement pattern parameters. Scaling the time parameters on the overall duration would reduce this level of complexity, however, it was not sufficiently successful. Therefore, introducing a second evaluation, once the feet have been brought to the bar, should be considered.

\section{REFERENCES}

Bartlett, R., Wheat, J., \& Robins, M. (2007). Is movement variability important for sports biomechanists? Sports Biomechanics, 6, 224-243. 
Begon, M., Wieber, P-B., \& Yeadon, M.R. (2008). Kinematics estimation of straddled movements on high bar from a limited number of skin markers using a chain model. Journal of Biomechanics, 41, 581-586.

Bevan, R., \& Corser T. (1969). Biomechanical study of gymnastic movements. The Gymnast, March, 30.

Carroll, D.L., 2001. FORTRAN genetic algorithm driver. Downloaded from: http://cuaerospace.com/carroll.ga.html

Fédération Internationale de Gymnastique. (2017). Code of Points. Moutier, Switzerland: F.I.G.

Forrester, S.E., Yeadon, M.R., King, M.A., \& Pain, M.T.G. (2011). Comparing different approaches for determining joint torque parameters from isovelocity dynamometer measurements. Journal of Biomechanics, 44, 955-961.

Fukushima, S., \& Russell, W. (1980). Men's Gymnastics. London: Faber \& Faber.

Grierson, L.E.M., \& Elliott, D. (2008). Kinematic analysis of goal-directed aims made against early and late perturbations: An investigation of the relative influence of two online control processes. Human Movement Science, 27, 839-856.

Gymnastics HQ. (2016). How to do a kip. https://gymnasticshq.com/how-to-do-a-kip/) Hamill, J., van Emmerik, R.E.A., Heiderscheit, B.C., \& Li, L. (1999). A dynamical systems approach to lower extremity running injuries. Clinical Biomechanics, 14, 297-308.

Hiley, M.J., Schmid, N., \& Yeadon, M.R. (2019). How do technique and coordination change during learning of a whole-body task: Application to the upstart in gymnastics. Journal of Sports Sciences, 37, 2374-2380.

Hiley, M.J. \& Yeadon, M.R. (2003). Optimum technique for generating angular momentum in accelerated backward giant circles prior to a dismount. Journal of Applied Biomechanics, 19, 119-130.

Hiley, M.J. \& Yeadon, M.R. (2012). Achieving consistent performance in a complex whole body movement: the Tkatchev on high bar. Human Movement Science, 31, 834-843.

Hiley, M.J., \& Yeadon, M.R. (2013). Investigating optimal technique in a noisy environment: application to the upstart on uneven bars. Human Movement Science, 32, 181-191.

Hiley, M.J., \& Yeadon, M.R. (2014). Determining the solution space for a co-ordinated whole body movement in a noisy environment: application to the upstart in gymnastics. Journal of Applied Biomechanics, 30, 508-513.

Hiley, M.J. and Yeadon, M.R. (2016). The role of functional variability in a whole body co-ordinated movement - application to high bar giant circles. Human Movement Science, 49, 95-103.

Jagacinski, R.J. \& Flach, J.M. (2003). Control Theory for Humans: Quantitative Approaches to Modeling Performance. Mahwah: Erlbaum.

James, C.R. (2004). Considerations of movement variability in biomechanical research. In N. Stergiou (Ed.), Innovative Analyses of Human Movement - 2004 (pp. 29-62). Champaign: Human Kinetics.

Lai, Q., Shea, C.H., Wulf, G. \& Wright, D.L. (2000). Optimizing generalized motor program parameter learning. Research Quarterly for Exercise and Sport, 71, 1024.

Miall, R.C., \& Wolpert, D.M. (1996). Forward models for physiological motor control. Neural Networks, 9, 1265-1279.

Newell, K.M. (2003). Schema theory (1975): Retrospectives and prospectives. Research Quarterly for Exercise and Sport, 74, 383-388. 
Preatoni, E., Hamill, J., Harrison, A.J., Hayes, K., Van Emmerik, R.E.A., Wilson, C., \& Rodano, R. (2013). Movement variability and skills monitoring in sports. Sports Biomechanics, 12, 69-92.

Readhead, L. (1987). Men's Gymnastics Coaching Manual. Huddersfield: Springfield Books.

Sayyah, M., Hiley, M.J., King M.A. \& Yeadon, M.R. (2018). Functional variability in the flight phase of one metre springboard forward dives. Human Movement Science, 59, 234-243.

Schmidt, R.A. (1975). A schema theory of discrete motor skill learning. Psychological Review, 82, 225-260.

Schmidt, R.A. (2003). Motor schema theory after 27 years: Reflections and implications for a new theory. Research Quarterly for Exercise and Sport, 74, 366-375.

Shapiro, D.C. (1977). A preliminary attempt to determine the duration of a motor program. In D.M. Landers \& R.W. Christina (Eds.), Psychology of Motor Behaviour and Sport - 1976 (pp. 17-24). Champaign: Human Kinetics.

Shea, C.H. \& Wulf, G. (2005). Schema Theory: A critical appraisal and reevaluation. Journal of Motor Behavior, 73, 85-102.

Summers, J.J. (1975). The role of timing in motor program representation. Journal of Motor Behavior, 7, 229-241.

van Beers, R.J., Haggard, P., \& Wolpert, D.M. (2004). The role of execution noise in movement variability. Journal of Neurophysiology, 91, 1050-1063.

Wulf, G., Lee, T.D., \& Schmidt, R.A. (1994). Reducing knowledge of results about relative versus absolute timing: Differential effects on learning. Journal of Motor behaviour, 26, 362-369.

Wolpert, D.M. (1997). Computational approaches to motor control. Trends in Cognitive Sciences, 1, 209-216.

Wood, G.A., \& Jennings, L.S. (1979). On the use of spline functions for data smoothing. Journal of Biomechanics, 12, 477-479.

Yamasaki, T., Gotoh, K., \& Xin, X. (2010). Optimality of a kip performance on the high bar: An example of skilled goal-directed whole body movement. Human Movement Science, 29, 464-482. 